\title{
Spatial frequency domain model for adaptive optics compensation of segmented mirror misalignments and figure errors
}

\section{Brent L. Ellerbroek}

Brent L. Ellerbroek, "Spatial frequency domain model for adaptive optics compensation of segmented mirror misalignments and figure errors," Proc. SPIE 6272, Advances in Adaptive Optics II, 62724K (27 June 2006); doi: $10.1117 / 12.669312$

Event: SPIE Astronomical Telescopes + Instrumentation, 2006, Orlando, Florida, United States 


\title{
Spatial frequency domain model for adaptive optics compensation of segmented mirror misalignments and figure errors
}

\author{
Brent L. Ellerbroek \\ Thirty Meter Telescope Project, 1200 E. California Blvd, MC 102-8, Pasadena, California
}

\begin{abstract}
In addition to their essential function of providing atmospheric turbulence compensation, astronomical Adaptive Optical ( $\mathrm{AO}$ ) systems also supplement the role of active optics $(\mathrm{aO})$ by providing some additional correction of the wavefront aberrations introduced by mirror mounting, alignment, thermal distortion and/or fabrication errors. This feature is particularly desirable for segmented mirror telescopes such as the Thirty Meter Telescope (TMT), but wavefront discontinuities across segment boundaries are challenging to properly sense and correct. In this paper we describe a fast, analytical, frequency domain model which may be used to study and quantify the above effects, and discuss a range of sample results obtained to support the development of the top-level requirements for the TMT primary mirror. In general, AO compensation of mirror segment piston errors is not particulary useful unless the deformable mirror (DM) interactuator spacing is equivalent to no more than one-half of a mirror segment diameter (when both of these dimensions are expressed in the same pupil plane). Effective AO compensation of mirror segment tip/tilt errors, or low order segment figure errors such as astigmatism, typically requires 3-4 DM actuators per mirror segment. These results illustrate the importance of quantifying and minimizing uncorrectable telescope wavefront errors when developing performance predictions for adaptive optical systems.
\end{abstract}

Keywords: Adaptive Optics, Extremely Large Telescopes, Segmented Mirrors

\section{INTRODUCTION}

The baseline design for the Thirty Meter Telescope (TMT) primary mirror consists of 738 hexagonal mirror segments, all of which must be properly fabricated, mounted, and aligned to precisely match the shape of the desired ellipsoidal optical prescription. ${ }^{1}$ A very high-order Shack-Hartmann wavefront sensor (WFS) will be used to initialize the rigid body alignment of the segments, and also to determine commands for the "warping harnesses" which will null the quadratic and cubic modes of the figure distortions induced by segment fabrication errors, mounting errors and quasi-static thermal distortion. ${ }^{2}$ Edge sensors will then monitor pointwise discontinuities between the segments, and the primary mirror control system (M1CS) will null these errors in closed loop. ${ }^{3}$

In spite of these calibration and control processes, residual wavefront errors will still be induced by higherorder mirror figure errors, temperature variations and gradients, edge sensor drift, and segment vibrations excited by the wind. It is of considerable interest to understand how these wavefront errors will impact the performance of the TMT adaptive optics (AO) systems, and investigate whether the AO systems themselves can compensate for at least some fraction of these effects.

Detailed simulations of TMT AO systems in the time domain are certainly feasible ${ }^{4}$ and are in many ways essential for developing detailed performance estimates and error budgets, but the amount of computer time required for these codes can limit their utility for performing trade studies and developing insight. We have therefore developed a fast, analytical, spatial-frequency-domain model to study the ability of the TMT AO systems to compensate for the wavefront aberrations induced by primary mirror segment figure and alignment errors. System performance is characterized in terms of the residual RMS wavefront error remaining after AO correction, which is an appropriate metric for the TMT natural guide star (NGS) AO and laser guide star (LGS)

Further author information: E-mail: brente@caltech.edu, Telephone: 16263951620

Advances in Adaptive Optics II, edited by Brent L. Ellerbroek, Domenico Bonaccini Calia, Proc. of SPIE Vol. 6272, 62724K, (2006) · 0277-786X/06/\$15 · doi: 10.1117/12.669312 
AO modes including multi-conjugate AO (MCAO), mid infra-red AO (MIRAO), ground-layer AO (GLAO), and multi-object AO (MOAO).$^{5}$ This analysis can be used to assist in the initial development of tolerances for the TMT primary mirror figure and alignment errors, although higher fidelity simulations will still be required to understand the higher-order impacts of primary mirror errors on certain AO modes in greater detail (For example, their effect on the image contrast achievable using an Extreme AO (ExAO) system. ${ }^{6}$ ).

Very briefly, the spatial frequency domain model presented in this paper assumes that segment fabrication errors can be modeled by "cookie cutting" hexagonal domains from infinite random phase screens with shiftinvariant statistics defined, for example, by a Komogorov spectrum or some other PSD. A number of low-order modes (piston, tip/tilt, astigmatism ....) are then removed from each segment to represent the process of initially aligning the segments and adjusting their warping harnesses. Note that this approach is unable to model many of the more complex characteristics of fabrication errors, for example edge roll-off. Next, segment misalignment errors are modeled as random linear combinations of tip/tilt and piston modes; segment figure distortions due to effects such as drift in the warping harness forces or temperature variations may also be simulated if higher-order modes (focus through astigmatism, or possibly trefoil) are included. These random errors are assumed to be zero mean, normally distributed, and have shift-invarient second-order statistics so that the correlations between errors on segments $i$ and $j$ depend only upon the center-to-center separation between the segments. Finally, the wavefront correction provided by the AO system is modeled as a spatial filtering operation. ${ }^{7}$ Since the primary mirror is the TMT entrance pupil, it is sufficient to consider the case of a conventional AO system with a single (Shack-Hartmann) WFS and a single deformable mirror optically conjugate to the primary. A WFS anti-aliasing filter $^{8}$ is included as a modeling option that eliminates the wavefront reconstruction errors induced by so-called "WFS spatial aliasing."

The current TMT specification for segment fabrication errors is based upon a Kolmogorov turbulence spectrum with an effective coherence diameter, $r_{0}$, of 1.0 meters at a wavelength of $0.5 \mu \mathrm{m}$. Under these assumptions, the principal conclusion of this study is that an $\mathrm{AO}$ system will only provide appreciable compensation of these errors if the WFS subaperture size is significantly smaller than 0.5 meters. This result is obtained with or without WFS spatial aliasing, and in fact performance (in terms of the residual RMS wavefront error) is not significantly degraded by the absence of the anti-aliasing filter. In contrast, an $\mathrm{AO}$ system with 0.5 meter subapertures does correct for a reasonable fraction of the effect of segment misalignments-about 60 per cent of the wavefront error is corrected for segment piston errors, and about 40 per cent for tip/tilt. These two results are in some sense consistent: segment figure errors are only marginally compensated by an order 60x60 AO system because the easily compensated tip/tilt/piston components of the error have already been corrected by segment alignment adjustments.

Section 2 below outlines the theory developed for this analysis. Section 3 describes one of the important elements of the numerical implementation of this theory, namely how to compute the Fourier transforms of low-order polynomial modes defined on hexagonal domains. The numerical results themselves are presented in Section 4 .

\section{MODELING APPROACH}

The segmented mirror wavefront aberrations to be studied in this report will be modeled using the equation

$$
\phi_{o}(r)=p(r-s)+q(r-s),
$$

where $\phi_{o}$ is the wavefront aberration before partial compensation by the AO system, the variable $r$ denotes 2-dimensional coordinates in the aperture plane, the function $p$ is the segmented mirror fabrication error with a number of low-order modes removed, and $q$ is the wavefront error contribution from segment misalignments and mounting distortions. The functions $p$ and $q$ are assumed to be zero-mean and statistically independent of each other.* Further details on the modeling of these two terms will be presented in the first two subsections below.

The variable $s$ denotes a random translation of the wavefront aberration $\phi_{0}$ relative to the WFS subaperture and DM actuator geometries in the AO system. Heuristically, this translation is included to eliminate any spatial

*If these errors are not zero mean, the effect of their mean value can be studied separately in a conventional AO simulation.

Proc. of SPIE Vol. 6272 62724K-2 
"beat frequencies" between the WFS/DM geometry and mirror segment geometry; how this feature simplfies the analysis will become clear in the following paragraphs. For AO systems on TMT and a variety of other telescopes, something very much like this effect will occur in one spatial dimension as the zenith angle of a science observation varies, and the image of the primary mirror rotates in the plane of a DM which is located on the telescope Nasmyth platform.

Using the Fourier shift theorem and the fact that $p$ and $q$ are statistically independent, the second-order statistics of the spatial Fourier transform of the wavefront aberration, $\hat{\phi}_{0}$, are given by the expression

$$
\begin{aligned}
\left\langle\hat{\phi}_{o}(\kappa) \hat{\phi}_{o}^{*}\left(\kappa^{\prime}\right)\right\rangle_{p, q, s} & =\left\langle\exp \left[-2 \pi i\left(\kappa-\kappa^{\prime}\right) \cdot s\right]\right\rangle_{s}\left[\left\langle\hat{p}(\kappa) \hat{p}^{*}\left(\kappa^{\prime}\right)\right\rangle_{p}+\left\langle\hat{q}(\kappa) \hat{q}^{*}\left(\kappa^{\prime}\right)\right\rangle_{q}\right] \\
& =\delta\left(\kappa-\kappa^{\prime}\right)\left[\left\langle\hat{p}(\kappa) \hat{p}^{*}\left(\kappa^{\prime}\right)\right\rangle_{p}+\left\langle\hat{q}(\kappa) \hat{q}^{*}\left(\kappa^{\prime}\right)\right\rangle_{q}\right] .
\end{aligned}
$$

Here $\kappa$ denotes a spatial frequency variable, the subscripted angle brackets, $\langle\ldots\rangle$, denote ensemble averaging over one or more random variables, ${ }^{\dagger}$ and the Dirac delta function is denoted as $\delta$. This formula demonstrates that the introduction of the random coordinate translation $s$ has effectively removed any cross-correlation between the value of $\hat{\phi}_{0}$ at distinct spatial frequencies $\kappa \neq \kappa^{\prime}$.

Our primary interest in this study is to evaluate the mean-square residual wavefront error $\sigma^{2}$ remaining after the aberration $\phi_{o}$ has been partially corrected by the AO system. We will assume that the DM actuators and WFS subapertures are arranged in an effectively infinite square grid with a spacing equal to $\Delta$, and that the DM actuator command vector is consequently obtained by applying a spatial filter (or convolution) to the WFS gradient measurement vector. The AO correction may potentially suffer from spatial aliasing because the WFS measurements are recorded on a discrete grid, so that the action of the AO system may be described in the spatial frequency domain by an equation of the form

$$
\hat{\phi}(\kappa)=\sum_{n} f\left(\kappa, \kappa+\Delta^{-1} n\right) \hat{\phi}_{o}\left(\kappa+\Delta^{-1} n\right) .
$$

Here the function $\phi$ is the residual wavefront aberration after AO compensation and $f$ denotes the error rejection transfer function of the AO system. The summation over multiple spatial frequencies in the formula reflects the potential impact of WFS spatial aliasing on the wavefront reconstruction process. The third subsection below provides further details upon the options for the error rejection function $f$, and general background information on Fourier domain modeling of AO systems is contained in an earlier paper.$^{7}$

Using the Plancheral theorem and Eq.'s (2) and (3) above, the mean-square residual wavefront error $\sigma^{2}$ may be written in the form

$$
\begin{aligned}
\sigma^{2}= & \left(N_{s} \Omega\right)^{-1}\left\langle\int d r \phi^{2}(r)\right\rangle \\
= & \left(N_{s} \Omega\right)^{-1}\left\langle\int d \kappa|\hat{\phi}(\kappa)|^{2}\right\rangle \\
= & \left(N_{s} \Omega\right)^{-1} \int d \kappa \sum_{n}\left|f\left(\kappa, \kappa+\Delta^{-1} n\right)\right|^{2} \\
& \quad \times\left[\left\langle\left|\hat{p}\left(\kappa+\Delta^{-1} n\right)\right|^{2}\right\rangle+\left\langle\left|\hat{q}\left(\kappa+\Delta^{-1} n\right)\right|^{2}\right\rangle\right],
\end{aligned}
$$

where $N_{s}$ is the number of segments in the mirror and $\Omega$ is the area of an individual segment. We note that this formula would not reduce to a single integral in the spatial frequency domain without the introduction of the random translation $s$, which would effectively eliminate most of the benefits of using a Fourier domain approach. Using a change of integration variable, this expression may be re-written as

$$
\sigma^{2}=\left(N_{s} \Omega\right)^{-1} \int d \kappa F(\kappa)\left[\left\langle|\hat{p}(\kappa)|^{2}\right\rangle+\left\langle|\hat{q}(\kappa)|^{2}\right\rangle\right]
$$

\footnotetext{
${ }^{\dagger}$ Subscripts will be used to denote the specific random variables when more than one option is available.
} 
where the function $F$ denotes the summation

$$
F(\kappa)=\sum_{n}\left|f\left(\kappa+\Delta^{-1} n, \kappa\right)\right|^{2} .
$$

Eq. (5) is the final general result for the residual mean-square phase error after AO compensation for generic segment figure errors, segment misalignments, and AO control algorithms. The following three subsections describe the models that will be used in this study for these quantities in greater detail.

\subsection{Segmented Mirror Fabrication Errors}

The segmented mirror fabrications errors are assumed to be statistically independent from segment to segment. The error on each segment is initially given as a "cookie cut" section of an infinite phase screen with shift-invariant statistics defined by a power spectrum, and the low-order modes of this error are then removed to simulate the process of aligning, and possibly warping, the segment into the telescope. This qualitative description is formalized in the paragraphs below.

The overall mirror fabrication error before low-order mode removal will be denoted as $P$, and is defined by the formula

$$
P(r)=\sum_{j} P_{j}\left(r-r_{j}\right) h\left(r-r_{j}\right)
$$

Here the summation variable $j$ runs over the segments in the mirror, $P_{j}$ is the fabrication error on segment $j$, $r_{j}$ denotes the center of this segment, and $h(r)$ is a $\{0,1\}$-valued function representing the clear aperture of a cannonical segment. The common area $\Omega$ of each segment is given by

$$
\Omega=\int d r h(r)
$$

Before "cookie cutting," the shift-invariant statistics of the segment phase errors are described by the expression

$$
\left\langle\hat{P}_{j}(\kappa) \hat{P}_{k}^{*}(\kappa)\right\rangle=\delta_{j k} \Phi(\kappa),
$$

where $\delta_{j k}$ is the Kroenecker delta and $\Phi$ is the power spectral density (PSD) of the fabrication errors, for example a Kolmogorov or von Karman spectrum.

Next, the process of correcting the low-order modes of the segment fabrication errors during the initial installation of the segments is modeled by the expression

$$
p(r)=P(r)-\Omega^{-1} \sum_{j, k} a_{j k} m_{k}\left(r-r_{j}\right),
$$

where $m_{k}$ are the low-order modes which are removed from each segment fabrication error and $a_{j k}$ is the coefficient of mode $k$ on segment $j$. The support of each mode $m_{j}$ is assumed to lie within the segment boundary, so that

$$
m_{j}(r)=m_{j}(r) h(r) .
$$

The modes are also assumed to be orthogonalized, with magnitudes scaled to satisfy the formula

$$
\int d r m_{j}(r) m_{k}(r)=\delta_{j k} \Omega
$$

Given these conditions, it is not difficult to derive that the modal coefficients yielding the best fit ${ }^{\ddagger}$ to the segment fabrication error $P$ is given by the expression

$$
a_{j k}=\int d r P_{j}(r) m_{k}(r)
$$

\footnotetext{
${ }^{\ddagger}$ Of course, these low-order modes will not be removed perfectly from the segment fabrication errors, but any residuals are assumed to be statistically independent of the initial fabrication error $P$, and they may therefore be treated as part of the segment misalignment and mounting error $q$ describe further below.
} 
It is now possible to derive the relationship

$$
\left\langle|\hat{p}(\kappa)|^{2}\right\rangle=N_{s} \int d \eta \Phi(\eta)\left|\hat{h}(\kappa-\eta)-\Omega^{-1} \sum_{j} \hat{m}_{j}(\kappa) \hat{m}_{j}^{*}(\eta)\right|^{2}
$$

for the frequency domain statistics of the fabrication errors after low-order mode removal. This formula resembles a convolution and would be inefficient to evaluate numerically, and so it is computationally simpler to work with the less elegant expression

$$
\begin{aligned}
N_{s}^{-1}\left\langle|\hat{p}(\kappa)|^{2}\right\rangle=\mathcal{F} & {\left[\mathcal{F}^{-1}(\Phi) \mathcal{F}^{-1}\left(|\hat{h}|^{2}\right)\right](\kappa) } \\
& -\Omega^{-1} \sum_{j} \hat{m}_{j}(\kappa) \mathcal{F}\left[\mathcal{F}^{-1}\left(\Phi \hat{m}_{j}^{*}\right) \mathcal{F}^{-1}\left(\hat{h}^{*}\right)\right](\kappa) \\
& -\Omega^{-1} \sum_{j} \hat{m}_{j}^{*}(\kappa) \mathcal{F}\left[\mathcal{F}^{-1}\left(\Phi \hat{m}_{j}\right) \mathcal{F}^{-1}(\hat{h})\right](\kappa) \\
& +\Omega^{-2} \sum_{j, k} \hat{m}_{j}(\kappa) \hat{m}_{k}^{*}(\kappa) \int d \eta \Phi(\eta) \hat{m}_{j}^{*}(\eta) \hat{m}_{k}(\eta) .
\end{aligned}
$$

Here $\mathcal{F}$ denotes the 2-dimentional spatial Fourier transfrom operator. Sample value of $\left\langle|\hat{p}(\kappa)|^{2}\right\rangle$ are presented in section 3 below for the case of hexagonal segments with a Kolmogorov spectrum for their fabrication errors.

\subsection{Segmented Mirror Alignment and Mounting Errors}

The wavefront aberration $q$ due to these two effects is somewhat easier to describe and evaluate. It is given as a sum of contributions on each segment,

$$
q(r)=\sum_{j} q_{j}\left(r-r_{j}\right)
$$

with the wavefront error on segment number $j$ represented as a linear combination of the same low-order modes $m_{k}$ introduced above:

$$
q_{j}(r)=\sum_{k} a_{j k} m_{k}(r)
$$

The modal coefficients are assumed to be zero mean,

$$
\left\langle a_{j k}\right\rangle=0,
$$

and to have shift-invariant second-order statistics described by the expression:

$$
\left\langle a_{j k} a_{j^{\prime} k^{\prime}}\right\rangle=C_{k k^{\prime}}\left(r_{j}-r_{j^{\prime}}\right) .
$$

The quantity $C(r)$ should be viewed as a collection of matrix-valued delta functions defined only at those points $r$ corresponding to separations between mirror segments.

Assuming the above model, we may evaluate the second-order statistics of the wavefront errors induced by segment misalignments using the expression

$$
\left\langle|\hat{q}(\kappa)|^{2}\right\rangle=\hat{m}^{*}(\kappa) \mathcal{C}(\kappa) \hat{m}(\kappa) .
$$

Here $\hat{m}(\kappa)$ is a vector with components equal to the Fourier transforms of the individual misalignment modes,

$$
\hat{m}(\kappa)=\left(\begin{array}{c}
\hat{m}_{1}(\kappa) \\
\vdots \\
\hat{m}_{n}(\kappa)
\end{array}\right)
$$


and the matrix $\mathcal{C}(\kappa)$ is defined by the formula

$$
\mathcal{C}(\kappa)=\sum_{j, j^{\prime}} \exp \left[-2 \pi i \kappa \cdot\left(r_{j}-r_{j^{\prime}}\right)\right] C\left(r_{j}-r_{j^{\prime}}\right)
$$

If (i) the number of segments in the mirror is large, and (ii) the covariance matrix $C(r)$ tends to zero rapidly enough for large separations $r$, it follows that the sum over $j^{\prime}$ in Eq. (22) will be approximately equal for the large majority of segments with locations $r_{j}$ located away from the boundary of the aperture. In the limit of an infinitely large segmented mirror telescope, the matrix $\mathcal{C}$ will therefore tend to the value

$$
\mathcal{C}(\kappa) \rightarrow N_{s} \hat{C}(\kappa)
$$

where the Fourier transform $\hat{C}$ of the covariance function $C$ is given by the expression

$$
\hat{C}(\kappa)=\sum_{j} \exp (-2 \pi i \kappa \cdot d) C(d),
$$

and the summation is performed over all 2-vectors $d$ which correspond to a separation between distinct mirror segments. If the alignment errors are in fact uncorrelated between different segments, the matrix $\mathcal{C}$ will take the simpler form

$$
\mathcal{C}(\kappa) \equiv N_{s} C(0)
$$

regardless of the number of segments in the mirror.

\subsection{Adaptive Optics Model}

The adaptive optics model is based upon an ideal Shack Hartmann wavefront sensor that measures the average gradients, or slopes, of a phase distortion profile over an infinite array of square subapertures whose width and spacing are equal to $\Delta$. If this WFS measurement, $s(r)$, were a continuous function of $r$, it would be related to the wavefront aberration $\phi_{o}(r)$ by a spatial filtering operation of the form

$$
\hat{s}(\kappa)=G(\kappa) \hat{\phi}(\kappa),
$$

where the spatial filter $G(\kappa)$ first differentiates the aberration $\phi_{o}$ with respect to $x$ and $y$, and then averages these derivatives over square subapertures:

$$
G(\kappa)=\left(\frac{\sin \pi \Delta \kappa_{x}}{\pi \Delta \kappa_{x}}\right)\left(\frac{\sin \pi \Delta \kappa_{y}}{\pi \Delta \kappa_{y}}\right)\left(\begin{array}{l}
2 \pi i \kappa_{x} \\
2 \pi i \kappa_{y}
\end{array}\right) .
$$

However, the WFS in fact samples the measurement vector $s_{d}(r)$ at a discrete grid of points of the form $r=\Delta n=$ $\Delta\left(n_{x}, n_{y}\right)$, so that its Fourier transform $\hat{s}_{d}(\kappa)$ is periodic in $\kappa$ with a unit cell defined by $\max \left(\kappa_{x}, \kappa_{y}\right) \leq 1 /(2 \Delta)$.

A new Shack-Hartmann WFS concept has recently been proposed to spatially filter the input wavefront and prevent aliasing of higher-spatial frequency terms into this passband. ${ }^{8}$ We therefore consider WFS measurement models with and without this anti-aliasing filter (AAF), defined by the pair of expressions

$$
\hat{s}_{d}(\kappa)= \begin{cases}\hat{s}(\kappa) & \mathrm{AAF}, \\ \sum_{n} \hat{s}\left(\kappa+\Delta^{-1} n\right) & \text { no AAF. }\end{cases}
$$

A classical least-squares pseudo-inverse wavefront reconstruction algorithm will be used with either of these measurement models. The phase estimate $\varphi(r)$ is given by

$$
\hat{\varphi}(\kappa)= \begin{cases}{\left[G^{*}(\kappa) G(\kappa)\right]^{-1} G^{*}(\kappa) s_{d}(\kappa)} & \text { if } \max \left\{\kappa_{x}, \kappa_{y}\right\} \leq 1 /(2 \Delta), \\ 0 & \text { otherwise. }\end{cases}
$$

From Eq. (28), it follows that this estimation algorithm accurately reconstructs those spatial frequency components of the phase distortion profile $\phi_{o}$ which lie within the passband of the WFS, provided that an anti-aliasing 
filter is utilized. The wavefront estimate is otherwise corrupted by an additive error term sometimes referred to as "WFS spatial aliasing noise."

In the notation of Eq. (3) above, the error rejection function $f$ of the AO system will always satisfy the condition

$$
f\left(\kappa_{f}, \kappa_{f}\right)=0
$$

for any "fundamental" frequency $\kappa_{f}$ with $\max \left\{\kappa_{f, x}, \kappa_{f, y}\right\} \leq 1 /(2 \Delta)$; for frequencies of the form $\kappa=\kappa_{f}+\Delta^{-1} n$ with $n=\left(n_{x}, n_{y}\right) \neq(0,0)$, the crosstalk in the wavefront reconstruction process is defined by

$$
f\left(\kappa_{f}, \kappa\right)= \begin{cases}0 & \mathrm{AAF} \\ {\left[G^{*}\left(\kappa_{f}\right) G\left(\kappa_{f}\right)\right]^{-1} G^{*}\left(\kappa_{f}\right) G(\kappa)} & \text { no AAF }\end{cases}
$$

and finally

$$
f\left(\kappa, \kappa^{\prime}\right)=\delta\left(\kappa-\kappa^{\prime}\right)
$$

for any two frequencies $\kappa$ and $\kappa^{\prime}$ lying outside the measurement passband for either WFS option, since the AO system does not apply a correction of any sort for such values of $\kappa$.

Combining Eq.'s (27) and (30)-(32) above, it follows that the function $F(\kappa)$ defined by Eq. (6) takes the values

$$
F\left(\kappa_{f}\right)=0,
$$

for any "fundamental" frequency $\kappa_{f}$ lying within the WFS spatial frequency passband, and

$$
F(\kappa)= \begin{cases}1 & \mathrm{AAF}, \\ 1+\left(\frac{\kappa_{f}^{T} \kappa}{\kappa_{f}^{T} \kappa_{f}}\right)^{2}\left(\frac{\kappa_{f, x}}{\kappa_{x}}\right)^{2}\left(\frac{\kappa_{f, y}}{\kappa_{y}}\right)^{2} & \text { no AAF }\end{cases}
$$

for any higher spatial frequency $\kappa$ given by $\kappa=\kappa_{f}+\Delta^{-1} n . \S$ This is the final result needed to evaluate the last of the three terms appearing in Eq. (5) for the phase variance $\sigma^{2}$ remaining after segmented mirror fabrication, alignment, and mounting errors have been partially compensated by the AO system.

\section{NUMERICAL IMPLEMENTATION}

The theory described above has been coded into a set of relatively modest MATLAB routines. This code evaluates Eq. (5) to determine the residual mean-square phase error $\sigma^{2}$ for the case of hexagonal mirror segments with von Karman fabrication errors, and misalignment and mounting error given in terms of a polynomial basis set. The set of input parameters required for the calculation is as follows:

- The segment width $d$;

- the equivalent coherence diameter $r_{0}$ and outer scale $L_{0}$ of the segment fabrication errors;

- the (radial) order of the low-order polynomial modes removed from the fabrication errors;

- the polynomial basis functions for the segment misalignment and mounting errors, and the misalignment error covariance matrix in terms of this basis;

- the WFS subaperture width $\Delta$; and

- the spacing and size of the Fourier domain numerical integration grid.

\footnotetext{
${ }^{\S}$ Here $x^{T}$ denotes the transpose of a vector $x$, so that $x^{T} y$ is the inner product of two vectors $x$ and $y$.
} 
The phase variance $\sigma^{2}$ is computed with and without the WFS anti-aliasing spatial filter for each parameter set.

Perhaps the most complex part of the implementation work was the development of a MATLAB routine to compute the Fourier transforms $\hat{m}_{j}(\kappa)$ of the low-order modes $m_{j}(r)$ appearing in the definition of the fabrication errors $p(r)$ and the segment misalignment error $q(r)$. The approach used is outlined briefly below.

Since all of the low-order modes considered in this analysis are polynomials, it is sufficient to develop a means of evaluating integrals of the form

$$
i_{n m}(\kappa)=\int d x d y h(x, y) x^{n} y^{m} \exp \left[-2 \pi i\left(\kappa_{x} x+\kappa_{y} y\right)\right],
$$

where $n$ and $m$ are non-negative integers. As illustrated in Fig. (1), the domain of integration defined by the $\{0,1\}$-valued hexagon function $h$ is actually the union of three parallelograms. The appropriate changes of variable enable the integral to be evaluated as the sum of three integrals on unit squares,

$$
i_{n m}(\kappa)=\sum_{j=1}^{3} \int_{0}^{1} \int_{0}^{1} d u d v\left|\frac{\partial\left(x_{j}, y_{j}\right)}{\partial(u, v)}\right| x_{j}^{n} y_{j}^{m} \exp \left[-2 \pi i\left(\kappa_{x} x_{j}+\kappa_{j} y_{j}\right)\right],
$$

where the coordinate transformations between $(u, v)$ and $(x, y)$ are defined as

$$
\begin{aligned}
& \left(\begin{array}{l}
x_{1} \\
y_{1}
\end{array}\right)=d\left(\begin{array}{cc}
1 / 2 & 0 \\
-1 /(2 \sqrt{3}) & 1 / \sqrt{3}
\end{array}\right)\left(\begin{array}{l}
u \\
v
\end{array}\right), \\
& \left(\begin{array}{l}
x_{2} \\
y_{2}
\end{array}\right)=d\left(\begin{array}{cc}
-1 / 2 & 0 \\
-1 /(2 \sqrt{3}) & 1 / \sqrt{3}
\end{array}\right)\left(\begin{array}{l}
u \\
v
\end{array}\right), \\
& \left(\begin{array}{l}
x_{3} \\
y_{3}
\end{array}\right)=d\left(\begin{array}{cc}
1 / 2 & -1 / 2 \\
-1 /(2 \sqrt{3}) & -1 /(2 \sqrt{3})
\end{array}\right)\left(\begin{array}{l}
u \\
v
\end{array}\right) .
\end{aligned}
$$

It is therefore sufficient to develop an algorithm for evaluating integrals of the form

$$
\begin{aligned}
I_{n, m}(\kappa ; A)= & \int_{0}^{1} \int_{0}^{1} d u d v\left|\begin{array}{cc}
a_{11} & a_{12} \\
a_{21} & a_{22}
\end{array}\right|\left(a_{11} u+a_{12} v\right)^{n}\left(a_{21} u+a_{22} v\right)^{m} \\
& \times \exp \left\{-2 \pi i\left[\left(a_{11} \kappa_{x}+a_{21} \kappa_{y}\right) u+\left(a_{21} \kappa_{x}+a_{22} \kappa_{y}\right) v\right]\right\} \\
= & \left|\begin{array}{cc}
a_{11} & a_{12} \\
a_{21} & a_{22}
\end{array}\right| \sum_{j=0}^{n} \sum_{k=0}^{m}\left(\begin{array}{c}
n \\
j
\end{array}\right)\left(\begin{array}{c}
m \\
k
\end{array}\right) a_{11}^{j} a_{12}^{n-j} a_{21}^{k} a_{22}^{m-k} \\
& \times \int_{0}^{1} d u u^{j+k} \exp \left[-2 \pi i\left(a_{11} \kappa_{x}+a_{21} \kappa_{y}\right) u\right] \\
& \times \int_{0}^{1} d v v^{n+m-j-k} \exp \left[-2 \pi i\left(a_{12} \kappa_{x}+a_{22} \kappa_{y}\right) v\right],
\end{aligned}
$$

where the second equality follows from the binomial expansion formula.

The single integral

$$
\mathcal{I}(n, \nu)=\int_{0}^{1} d u u^{n} \exp (-2 \pi i u \nu)
$$

appearing in Eq. (40) may now be evaluated via recursion. Using integration by parts we obtain

$$
\begin{aligned}
\mathcal{I}(0, \nu) & = \begin{cases}1 & \text { if } \nu=0, \\
\frac{1-\exp (2 \pi i \nu)}{2 \pi i \nu} & \text { otherwise, }\end{cases} \\
\mathcal{I}(n+1, \nu) & = \begin{cases}(n+2)^{-1} & \text { if } \nu=0, \\
\left(\frac{n+1}{2 \pi i \nu}\right) \mathcal{I}(n, \nu)-\frac{\exp (-2 \pi i \nu)}{2 \pi i \nu} & \text { otherwise. }\end{cases}
\end{aligned}
$$

This recursion relation is easily evaluated in MATLAB for small to moderate values of $n$. 


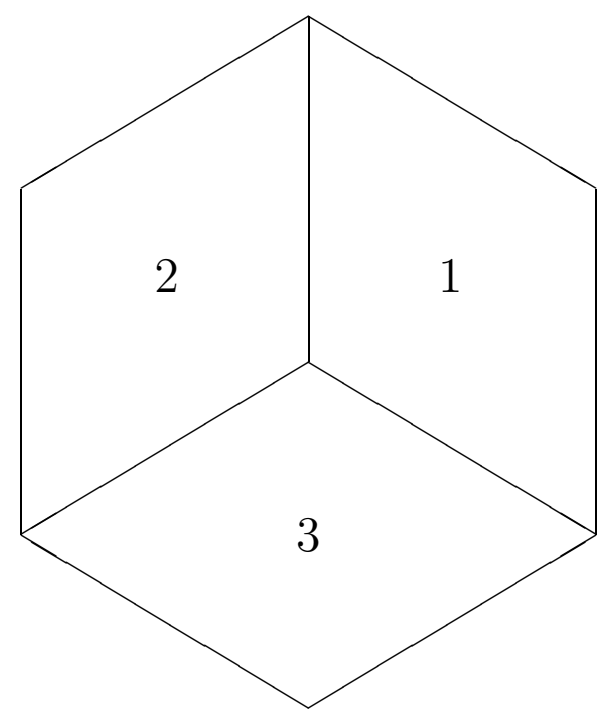

Figure 1. The integers indicate the domains of the three coordinate transformations defined by Eq.'s (37) through (39) in section 3.

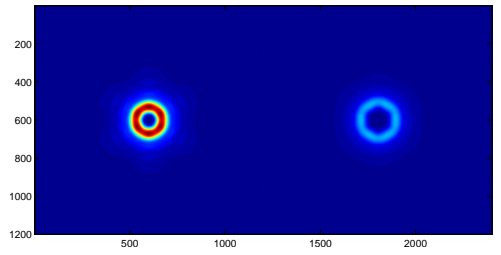

(a) Common color scale

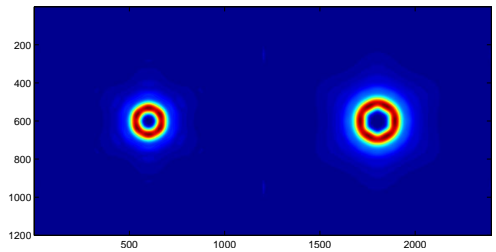

(b) Separate color scales

Figure 2. These figures illustrate the fabrication error power spectrum $\left\langle|\hat{p}(\kappa)|^{2}\right\rangle$ with low order modes removed up to either tip and tilt (left) or astigmatism (right). These results were obtained for the case of a hexagonal segment with a corner-to-corner spacing of 1.2 meters, and a Kolmogorov figure error spectrum with an $r_{0}$ of 1.0 meters. The figures illustrate that the total power in the spectrum decreases as the number of modes removed from the figure error increases, but the spatial frequency of the remaining error also increases.

The function $\mathcal{I}(n, \nu)$ may now be evaluated numerically on grids of values of $\nu_{j}$ of the form $\left(a_{1 j} \kappa_{x}+a_{2 j} \kappa_{y}\right)$. The results obtained may then be substituted back into Eq.'s (40) and (36) to determine the Fourier transforms of the monomial $x^{n} y^{m}$ defined on the hexagonal segment $h$.

Fig. 2 illustrates samples value of the segment fabrication error term $\left\langle|\hat{p}(\kappa)|^{2}\right\rangle$ obtained using these methods. 


\section{SAMPLE NUMERICAL RESULTS}

The theory developed in the preceeding sections has been applied to a range of sample problems related to TMT. For segment fabrication errors, we have considered a segment corner-to-corner diameter of 1.2 meters, fabrication errors defined by a Kolmogorov spectrum with an $r_{0}$ of 1.0 meter at a wavelength of $\lambda=0.5 \mu \mathrm{m}$ (the current segment polishing specification), and low-order mode removal up to and including either zeroeth, first, or second-order radial modes. WFS subapertures sizes $\Delta$ of 1.0, 0.5 , and 0.25 meters have been considered (and also the case of no AO compensation at all), either with or without a WFS anti-aliasing filter.

Table 1 summarizes the residual RMS wavefront errors computed for this set of segment fabration error and AO system parameters. Some attenuation of piston-removed fabrication errors is obtained by an AO system with 0.5 meter subapertures, but in general, wavefront sensing and correction at a scale of 0.25 meters is required for a meaningful degree of fabrication error compensation. The RMS wavefront error values are only modestly reduced by the introduction of a WFS anti-aliasing filter, although this feature remains important for extreme AO (ExAO) systems more concerned with contrast ratios than simply the residual RMS wavefront error by itself.

Table 2 summarizes the results obtained for AO correction of the low-order wavefront aberrations introduced by segment misalignments and modal figure distortions. In this case, results are presented in terms of "sensitivities," defined as the ratio between the RMS wavefront errors before and after AO compensation for a particular misalignment mode. An AO system with 0.5 meter subapertures corrects for all but approximately 40 per cent of random piston errors; for first order tip/tilt modes the relative residual error increases to about 60 per cent, and to about 80 to 100 per cent for the quadratic error terms. All of these values improve when the WFS subaperture size is reduced to 0.25 meters, particularly for the quadratic modes.

\section{ACKNOWLEDGMENTS}

The author gratefully acknowledges the support of the TMT partner institutions. They are the Association of Canadian Universities for Research in Astronomy (ACURA), the Association of Universities for Research in Astronomy (AURA), the California Institute of Technology and the University of California. This work was supported, as well, by the Canada Foundation for Innovation, the Gordon and Betty Moore Foundation,

\footnotetext{
"Note that the error computed with piston errors removed (only) and no AO correction is underestimated by approximately 20 per cent, since in this case the fabrication error spectrum $\left\langle|\hat{p}(\kappa)|^{2}\right\rangle$ contains an integrable singularity of the form $|\kappa|^{-5 / 3}$ which is not accurately evaluated using the numerical integration routines developed for this study. The remainder of the values in the table are accurate at the level of relative errors of a few per cent, based upon numerical experiments in varying the size and spacing of the Fourier domain integration grids.
}

Table 1. Adaptive optics compensation of segmented mirror fabrication errors. This table lists the residual RMS wavefront error, in nanometers, after segmented mirror fabrication errors have been partially compensated by an AO system. This result depends upon the WFS subaperture size $\Delta$, whether or not an anti-aliasing filter (AAF) is include in the WFS design, and the radial order $O_{m}$ of low-order modes removed from the segment fabrication errors before AO compensation. All results assume a segment corner-to-corner width of 1.2 meters, an $r_{0}$ of 1 meter for the fabrication errors, and an infinite outer scale $L_{0}$.

\begin{tabular}{|c|c||c|c|c|c|}
\hline \multirow{2}{*}{$O_{m}$} & \multicolumn{1}{|c||}{ AAF } & \multicolumn{4}{|c|}{$\Delta, \mathrm{m}$} \\
\cline { 3 - 6 } & & No AO & 1.00 & 0.50 & 0.25 \\
\hline \multirow{2}{*}{0} & No & 72.7 & 74.6 & 51.5 & 31.9 \\
\cline { 2 - 6 } & Yes & 72.7 & 67.9 & 45.1 & 28.7 \\
\hline \multirow{2}{*}{1} & No & 30.0 & 32.2 & 29.9 & 18.6 \\
\cline { 2 - 6 } & Yes & 30.0 & 29.8 & 26.4 & 16.2 \\
\hline \multirow{2}{*}{2} & No & 20.8 & 22.1 & 22.3 & 15.9 \\
\cline { 2 - 6 } & Yes & 20.8 & 20.8 & 20.2 & 13.8 \\
\hline
\end{tabular}


Table 2. Adaptive optics compensation of segmented mirror alignment errors. This table lists the relative residual RMS wavefront error after each of the first six segmented mirror alignment modes have been partially compensated by an AO system. This result depends upon the WFS subaperture size $\Delta$, and whether or not an anti-aliasing filter (AAF) is include in the WFS design. As in Table 1, all results assume a segment corner-to-corner width of 1.2 meters. Note that the coefficient 0.15 appearing in the definition of the first quadratic mode yields a focus-like mode which is orthogonal to segment piston.

\begin{tabular}{|c|c||c|c|c|}
\hline \multirow{2}{*}{ Mode } & \multicolumn{1}{|c||}{ AAF } & \multicolumn{3}{c|}{$\Delta, \mathrm{m}$} \\
\cline { 3 - 5 } & & 1.00 & 0.50 & 0.25 \\
\hline \multirow{3}{*}{1} & No & 0.74 & 0.43 & 0.31 \\
\cline { 2 - 5 } & Yes & 0.64 & 0.39 & 0.27 \\
\hline \multirow{2}{*}{$x$} & No & 1.02 & 0.65 & 0.46 \\
\cline { 2 - 5 } & Yes & 0.92 & 0.56 & 0.38 \\
\hline \multirow{2}{*}{$y$} & No & 1.01 & 0.64 & 0.41 \\
\cline { 2 - 5 } & Yes & 0.92 & 0.57 & 0.38 \\
\hline$x^{2}+y^{2}-0.15$ & No & 1.07 & 0.92 & 0.56 \\
\cline { 2 - 5 } & Yes & 0.99 & 0.80 & 0.49 \\
\hline \multirow{3}{*}{$x y$} & No & 1.02 & 0.81 & 0.50 \\
\cline { 2 - 5 } & Yes & 0.99 & 0.76 & 0.46 \\
\hline$x^{2}-y^{2}$ & No & 1.13 & 1.03 & 0.61 \\
\cline { 2 - 5 } & Yes & 0.99 & 0.85 & 0.51 \\
\hline
\end{tabular}

the National Optical Astronomy Observatory, which is operated by AURA under cooperative agreement with the National Science Foundation, the Ontario Ministry of Research and Innovation, and the National Research Council of Canada.

\section{REFERENCES}

1. J. E. Nelson and G. H. Sanders, "TMT status report," in Ground-based and Airborne Telescopes, L. M. Stepp, ed., Proc. SPIE 6267, to appear.

2. G. A. Chanan, M. Troy, J. E. Nelson, and T. E. Mast, "The alignment and phasing system for the Thirty Meter Telescope," in Ground-based and Airborne Telescopes, L. M. Stepp, ed., Proc. SPIE 6267, to appear.

3. T. E. Mast, G. A. Chanan, J. E. Nelson, R. H. Minor, and R. C. Jared, "An edge sensor design for the Thirty Meter Telescope," in Ground-based and Airborne Telescopes, L. M. Stepp, ed., Proc. SPIE 6267, to appear.

4. L. Gilles, B. L. Ellerbroek, and J. Veran, "Laser guide star multi-conjugate adaptive optics performance of the Thirty Meter Telescope with elongated beacons and matched filtering," in Advances in Adaptive Optics II, B. L. Ellerbroek and D. B. Callia, eds., Proc. SPIE 6272, to appear.

5. B. L. Ellerbroek, C. Boyer, M. C. Britton, S. Browne, R. A. Buchroeder, M. K. Cho, M. R. Chun, R. Clare, L. G. Daggert, R. G. Dekany, J. H. Elias, D. A. Erickson, R. Flicker, D. T. Gavel, L. Gilles, G. Herriot, M. R. Hunten, R. R. Joyce, M. Liang, B. A. Macintosh, I. P. Powell, S. C. Roberts, E. Ruch, J. Sinquin, M. J. Smith, J. A. Stoesz, M. Troy, G. A. Tyler, J. Veran, C. R. Vogel, and Q. Yang, "A conceptual design for the Thirty Meter Telescope adaptive optics systems," in Advances in Adaptive Optics II, B. L. Ellerbroek and D. B. Callia, eds., Proc. SPIE 6272, to appear.

6. B. A. Macintosh, M. Troy, R. Doyon, K. L. Baker, J. R. Graham, and E. Serabyn, "Extreme adaptive optics for the Thirty Meter Telescope," in Advances in Adaptive Optics II, B. L. Ellerbroek and D. B. Callia, eds., Proc. SPIE 6272, to appear.

7. B. L. Ellerbroek, "Linear systems modeling of adaptive optics in the spatial frequency domain," J. Opt. Soc. Am. A 22, pp. 310-322, 2005. 
8. L. A. Poyneer and B. Macintosh, "Spatially filtered wavefront sensor for high-order adaptive optics," J. Opt. Soc. Am. A 21, pp. 810-819, 2004. 